\title{
NLRP3-mediated pyroptosis aggravates pressure overload-induced cardiac hypertrophy, fibrosis, and dysfunction in mice: cardioprotective role of irisin
}

\author{
Rongchuan Yue ${ }^{1}$, Zaiyong Zheng ${ }^{1}$, Yu Luo ${ }^{2}$, Xiaobo Wang ${ }^{1}$, Mingming LV', Dan Qin ${ }^{1}$, Qingqing Tan ${ }^{3}$, Yulong Zhang ${ }^{4}$, \\ Tao Wang ${ }^{5}$ and Houxiang $\mathrm{Hu}^{1}$
}

\begin{abstract}
The exact mechanism of myocardial hypertrophy has not been completely elucidated. NOD-like receptor protein 3 (NLRP3) and the pyroptotic cascade play a critical role in cardiac hypertrophy and inflammation. The myokine irisin can inhibit NLRP3 activation, although its exact mechanism of action is unknown. In this study, we induced cardiac hypertrophy in a mouse model via aortic constriction (TAC) to further explore the pathological role of NLRP3 inflammasome-mediated pyroptosis and the potential therapeutic effects of irisin. Cardiac hypertrophy significantly increased the percentage of apoptotic cells and upregulated IL-1 $\beta$, cleaved caspase-1, and GSDMD-N that lie downstream of the NLRP3 inflammasome. Subsequently, irisin was co-administered to the TAC mice or angiotensin II (Ang-II)-treated cardiomyocytes to observe whether it could attenuate pyroptosis and cardiac hypertrophy. We established a direct association between pyroptosis and cardiac hypertrophy and found that pharmacological or genetic inhibition of NLRP3 attenuated cardiac hypertrophy. Furthermore, ectopic overexpression of NLRP3 abrogated the cardioprotective effects of irisin. To summarize, pyroptosis is a pathological factor in cardiac hypertrophy, and irisin is a promising therapeutic agent that inhibits NLRP3-mediated pyroptosis of cardiomyocytes.
\end{abstract}

\section{Introduction}

Heart failure is a chronic condition ${ }^{1}$ that results from cardiac remodeling due to heightened damage or workload, heart damage due to pressure or volume overload, myocardial infarction, inflammatory heart muscle disorder, or idiopathic dilated cardiomyopathy ${ }^{2,3}$. Pathological hypertrophy of the myocardium is a critical risk factor of heart failure ${ }^{4}$ and frequently associated with

\footnotetext{
Correspondence: Tao Wang (157711435@qq.com) or

Houxiang Hu (hhxiangl7@163.com)

'Department of Cardiology, Affiliated Hospital of North Sichuan Medical College, 637000 Nanchong, P.R. China

${ }^{2}$ CSSD, Affiliated Hospital of North Sichuan Medical College, 637000 Nanchong, P.R. China

Full list of author information is available at the end of the article

These authors contributed equally: Rongchuan Yue, Zaiyong Zheng, Yu Luo Edited by Inna Lavrik
}

adverse events despite treatment options ${ }^{5,6}$. Therefore, it is necessary to elucidate the pathophysiological mechanisms underlying cardiac hypertrophy and develop novel therapeutic strategies.

Inflammasomes are pattern-recognition receptors that mediate the inflammatory response against pathogens and other stimuli. The NOD-like receptor protein 3 (NLRP3) inflammasome consists of NLRP3, caspase-1 (cysteinyl aspartate-specific proteinase-1), and ASC (apoptosisassociated speck-like protein). Following activation, NLRP3 recruits and cleaves pro-caspase-1, which triggers the pyroptotic cascade. The NLRP3 inflammasome is activated during several cardiac disorders, such as myocardial infarction, aortic valve diseases, myocarditis, ischemia/reperfusion injury, hypertension, and atherosclerosis $^{7}$, which is consistent with the increased

\section{(c) The Author(s) 2021}

(c) (i) Open Access This article is licensed under a Creative Commons Attribution 4.0 International License, which permits use, sharing, adaptation, distribution and reproduction cc) in any medium or format, as long as you give appropriate credit to the original author(s) and the source, provide a link to the Creative Commons license, and indicate if changes were made. The images or other third party material in this article are included in the article's Creative Commons license, unless indicated otherwise in a credit line to the material. If material is not included in the article's Creative Commons license and your intended use is not permitted by statutory regulation or exceeds the permitted use, you will need to obtain permission directly from the copyright holder. To view a copy of this license, visit http://creativecommons.org/licenses/by/4.0/. 


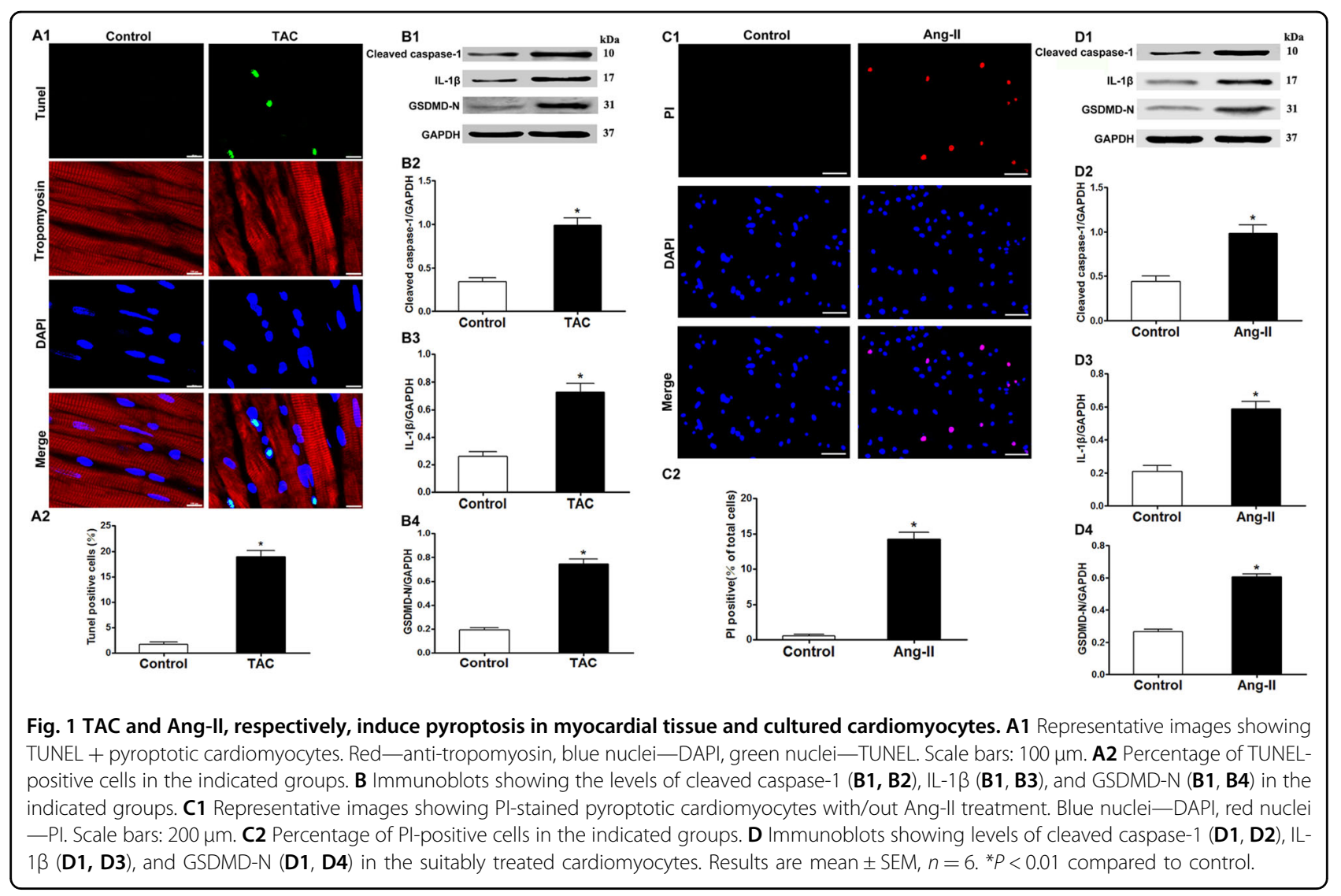

inflammation observed in stress-stimulated cardiac remodeling 8,9 .

Pyroptosis is a pro-inflammatory type of programmed cell death that depends on caspase- 1 or caspase-11 stimulation $^{10,11}$ and is morphologically and biochemically similar to necrosis and apoptosis. Unlike the other forms of programmed cell death, however, pyroptosis culminates in the secretion of pro-inflammatory cytokines ${ }^{12}$ and is associated with increased membrane porosity, cellular expansion, and DNA damage ${ }^{13}$. A recent study showed that NLRP3 inflammasome-mediated pyroptosis is a pathological factor underlying cardiovascular disease ${ }^{14}$, although its involvement in cardiac hypertrophy is unknown.

Irisin is a PGC1- $\alpha$-dependent myokine derived from the extracellular regions of fibronectin type III domaincontaining protein 5 (FNDC5) and converts white adipose tissue to brown adipose tissue $\mathrm{e}^{15}$. It also mitigates nondiabetic obesity and T2DM symptoms by inhibiting inflammation $^{16,17}$. Furthermore, recent studies have implicated a functional role of irisin in cardiovascular diseases, such as atherosclerosis ${ }^{18}$, myocardial ischemia/ reperfusion damage, and hypertension ${ }^{19,20}$. It remains to be elucidated whether irisin can ameliorate cardiac hypertrophy and heart failure by inhibiting NLRP3mediated pyroptosis. To this end, we established in vivo and in vitro models of cardiac hypertrophy and analyzed the effects of irisin on pressure overload-stimulated cardiac hypertrophy and NLRP3-mediated pyroptosis.

\section{Results}

Pressure overload causes pyroptosis in the heart tissue and cultured cardiomyocytes

A cardinal feature of pyroptosis is rapid membrane disintegration, which can be assessed with membraneimpermeable dyes, such as fluorescein-dUTP and $\mathrm{PI}^{21,22}$. As shown in Fig. 1A, the heart tissues from mice with cardiac hypertrophy had a significantly higher proportion of TUNEL + apoptotic cells compared to that from shamoperated controls. Consistent with this, the levels of cleaved caspase-1 (Fig. 1B1, B2) and IL-1 $\beta$ (Fig. 1B1, B3) were also upregulated in the myocardium of the TAC group relative to the control group. Furthermore, the specific pyroptosis marker GSDMD-N was also upregulated in the TAC group compared to the controls (Fig. 1B1, B4). Consistent with the in vivo results, the Ang-II-treated cardiomyocytes also showed significantly higher pyroptosis rates compared to the untreated cells, as indicated by the increased percentage of PI-positive cells (Fig. 1C), and upregulation of cleaved caspase-1 (Fig. 1D1, D2), IL-1 $\beta$ (Fig. 1D1, D3), and GSDMD-N (Fig. 1D1, D4) in the former. Taken together, pressure overload on cardiomyocytes induces pyroptosis. 

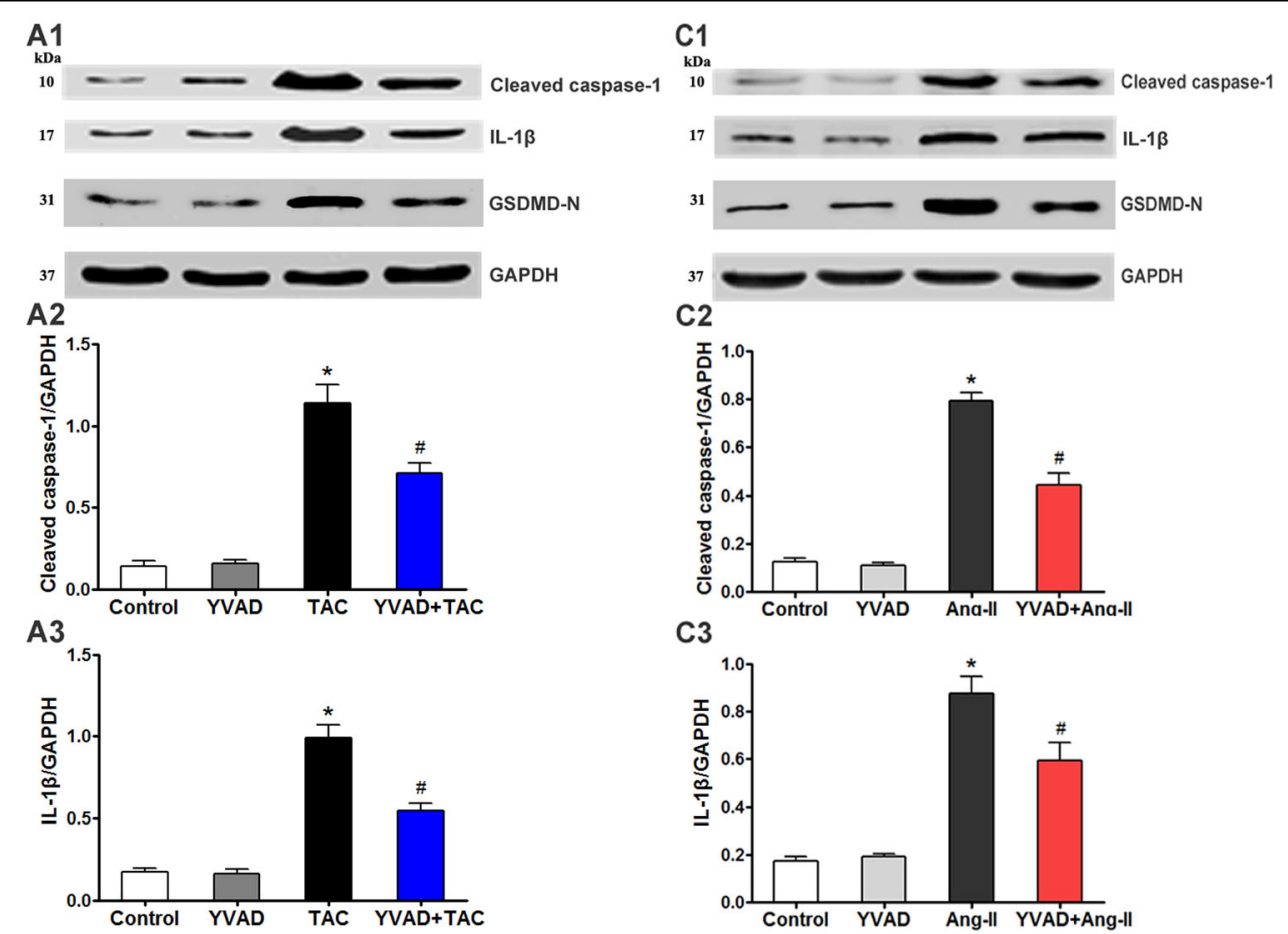

C3
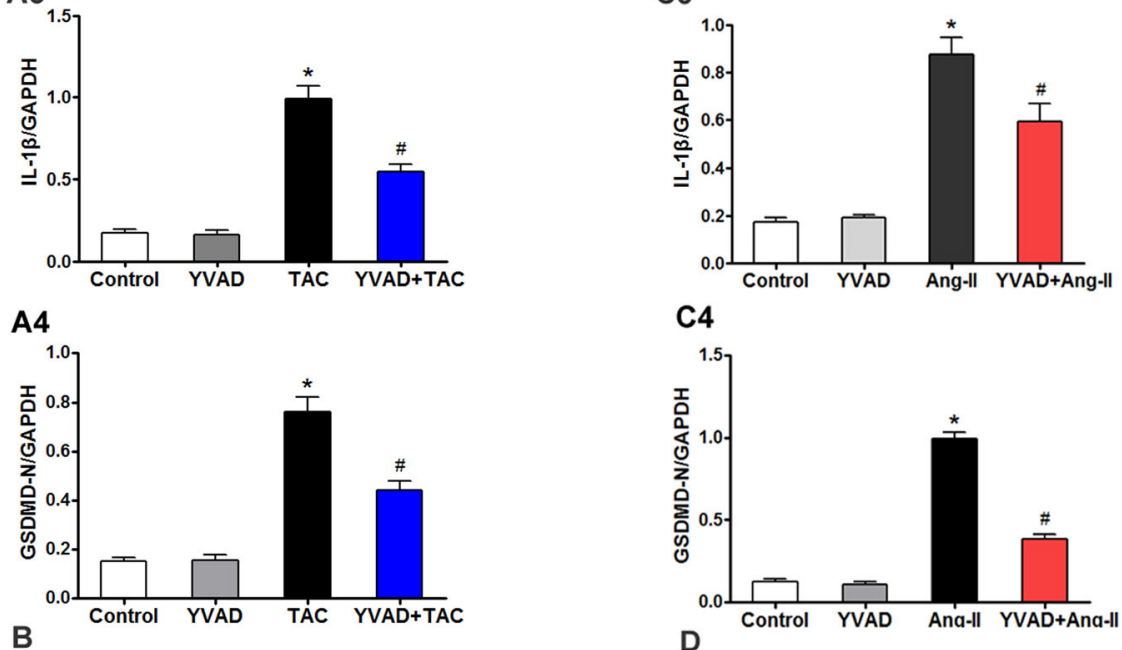

C4
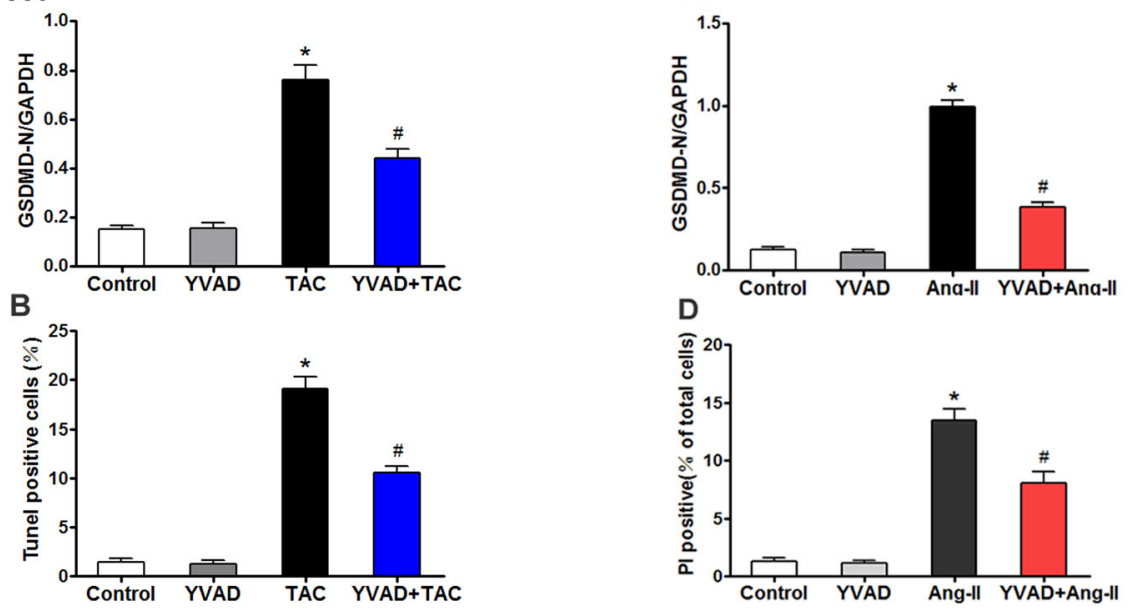

Fig. 2 Caspase-1 inhibition downregulates overload-induced pyroptosis. A Immunoblots showing levels of cleaved caspase-1 (A1, A2), IL-1 $\beta$ $(\mathbf{A} \mathbf{1}, \mathbf{A} 3)$, and GSDMD-N $(\mathbf{A} \mathbf{1}, \mathbf{A} 4)$ in the indicated groups. B Percentage of TUNEL-positive cells in the indicated groups. $\mathbf{C}$ Immunoblots showing levels of cleaved caspase-1 (C1, C2), IL-1 $3(\mathbf{C 1}, \mathbf{C} 3)$, and GSDMD-N $(\mathbf{C 1}, \mathbf{C} 4)$ in the suitably treated cardiomyocytes. D Percentage of PI-positive cells in the indicated groups. Results are mean $\pm \mathrm{SEM}, n=6 .{ }^{*} P<0.01$ compared to control, ${ }^{*} P<0.01$ compared to TAC group or Ang-\|l group. YVAD (ACYVAD-CMK, caspase-1 inhibitor).

\section{Caspase-1 inhibition downregulates pressure overload- induced pyroptosis}

To establish the potential correlation between pyroptosis and cardiac pressure overload, we analyzed the effects of caspase-1 inhibition on pressure overloadstimulated pyroptosis. The sham-operated and TAC mice were additionally treated with the caspase- 1 inhibitor AC-YVAD-CMK (YVAD), which not only mitigated the high levels of cleaved caspase-1 (Fig. 2A1, A2), IL-1 $\beta$
(Fig. 2A1, A3), and GSDMD-N (Fig. 2A1, A4) induced by TAC but also decreased the percentage of TUNELpositive cells (Fig. 2B). Likewise, cardiomyocytes cotreated with YVAD and Ang-II expressed lower levels of cleaved caspase-1 (Fig. 2C1, C2), IL-1 $\beta$ (Fig. 2C1, C3), and GSDMD-N (Fig. 2C1, C4) compared to the Ang-II-treated cells. In addition, YVAD also decreased the percentage of PI-positive cells in the presence of Ang II (Fig. 2D). Thus, YVAD mitigates pressure overload-induced pyroptosis in 
cardiomyocytes, indicating that activation of pyroptosis is directly related to pressure overload.

\section{Caspase-1 inhibition attenuates overload-stimulated cardiac hypertrophy}

To clarify the relationship between pyroptosis and cardiac hypertrophy, we analyzed the effect of YVAD on overload-stimulated cardiac hypertrophy, fibrosis, and heart failure. TAC significantly increased the cardiac mass as well as the proportion of heart weight (HW) to body weight (BW), suggesting that pressure overload led to cardiac hypertrophy (Fig. 3A). While YVAD significantly repressed the TAC-induced increase in HW/BW ratio, it had no effect on the healthy heart. Furthermore, YVAD also inhibited the increase in mean cardiomyocyte surface area following TAC (Fig. 3B). Pathological myocardial hypertrophy often leads to myocardial fibrosis, which is one of the mechanisms underlying heart failure. The mice hearts showed significant interstitial fibrosis and collagen accumulation 4 weeks after TAC (Fig. 3C). YVAD had no influence on the sham-operated hearts but significantly reduced the fibrotic area in the myocardium after TAC (Fig. 3C). Furthermore, the echocardiographic assessment indicated that TAC decreased the EF, and while YVAD significantly alleviated cardiac dysfunction, it had no major effect on the cardiac function of the sham-operated mice (Fig. 3D2). TAC mice showed a substantial upregulation in LVEDd (Fig. 3D3) and LVEDs (Fig. 3D4), which were significantly reversed by YVAD. The LVPWd of the hearts had thickened 4 weeks after TAC and was controlled by YVAD (Fig. 3D5). Thus, YVAD prevented TAC-induced cardiac dysfunction and alteration in ventricular structure. In addition, the survival rate of mice that underwent TAC was $63.6 \%$ at 2 weeks post surgery and dropped further to $45.5 \%$ at 4 weeks, which was consistent with the cardiac dysfunction induced by TAC. However, YVAD therapy sustained the survival rate at $81.8 \%$ at both 2 and 4 weeks after TAC (Fig. 3E). YVAD also significantly decreased the surface area of the Ang-IItreated cardiomyocytes in vitro (Fig. 3F1, F2), which correlated with the downregulation of hypertrophyrelated markers, such as brain natriuretic peptide (BNP) (Fig. 3G) and $\beta$-myosin heavy chain ( $\beta$-MHC) (Fig. 3H). Taken together, the activation of pyroptosis is correlated with cardiac hypertrophy, and its inhibition can attenuate overload-stimulated cardiac hypertrophy and heart failure.

\section{Irisin attenuates pressure overload-induced pyroptosis and cardiac hypertrophy}

To determine whether the NLRP3 inflammasome is stimulated by cardiac overload, the sham-operated and TACmodeled mice were treated with irisin. TAC-induced increase in NLRP3 (Fig. 4A1, A2), ASC (Fig. 4A1, A3), cleaved caspase-1 (Fig. 4A1, A4), and GSDMD-N (Fig. 4A1, A5) protein levels in the cardiac tissues were significantly reduced by irisin treatment. Co-treatment with irisin also decreased the percentage of TUNEL-positive cells compared with the untreated TAC group (Fig. 4B). Consistent with this, irisin downregulated the Ang-II-induced increase in NLRP3 (Fig. 4G1, G2), IL-1 3 (Fig. 4G1, G3), cleaved caspase-1 (Fig. 4G1, G4), and GSDMD-N (Fig. 4G1, G5) in the cardiomyocytes, and also decreased the percentage of PI-positive cells (Fig. 4H). These results indicate that the NLRP3 inflammasome is directly associated with pressure overload-induced pyroptosis in the cardiomyocytes. The enhanced HW/BW ratio in TAC mice was also significantly suppressed by irisin, which did not affect the healthy heart (Fig. 4C). Furthermore, irisin prevented the TAC-induced increase in the mean surface area of cardiomyocytes (Fig. 4D) and significantly reduced the myocardial fibrotic area (Fig. 4E). As shown in Fig. 4F, irisin significantly alleviated cardiac dysfunction caused by TAC but had no drastic influence on the cardiac function of the sham-operated mice. In addition, the cardiomyocytes co-treated with irisin and Ang II had lower surface area and reduced expression of BNP (Fig. 4J) and $\beta$-MHC (Fig. 4K) compared to those stimulated with Ang-II. Taken together, irisin mitigated pressure overloadinduced pyroptosis, as well as cardiac hypertrophy and fibrosis, by targeting the NLRP3 inflammasome.

\section{Inhibition of NLRP3 inflammasome alleviates pyroptosis and overload-stimulated cardiac hypertrophy}

The NLRP3 inflammasome is activated in various inflammatory disorders ${ }^{23-25}$. TAC significantly upregulated the NLRP3 (Fig. 5A1, A2), ASC (Fig. 5A1, A3), cleaved caspase-1 (Fig. 5A1, A4), and GSDMD-N (Fig. 5A1, A5) proteins in the myocardium, and increased the number of TUNEL-positive cells (Fig. 5B). Cytokine release inhibitory drug 3 (CRID3) $(200 \mathrm{mg} / \mathrm{kg} /$, dosed twice a day by oral gavage), a selective inhibitor of the NLRP3 inflammasome, reversed the TAC-induced pyroptosis cascade. Consistent with this, siRNA-mediated knockdown of NLRP3 in the Ang-II-treated cardiomyocytes decreased expression levels of NLRP3, ASC (Fig. 5G1, G2), cleaved caspase-1, and GSDMD-N (Fig. 5G1, G3), as well the number of PI-positive cells (Fig. 5H). CRID3 prevented the TAC-induced elevation in $\mathrm{HW} / \mathrm{BW}$ ratio but did not affect the mass of the normal heart (Fig. 5C). It also reversed the increase in mean cardiomyocyte surface area in the TAC mice (Fig. 5D), significantly decreased the myocardial fibrotic area (Fig. 5E), and normalized the EF (Fig. 5F). NLRP3-knockdown cardiomyocytes treated with Ang II had a lower surface area (Fig. 5I), and decreased levels of BNP and $\beta$-MHC (Fig. 5J) compared to the normal Ang-II-stimulated cells. Thus, inhibition of the NLRP3 inflammasome can 


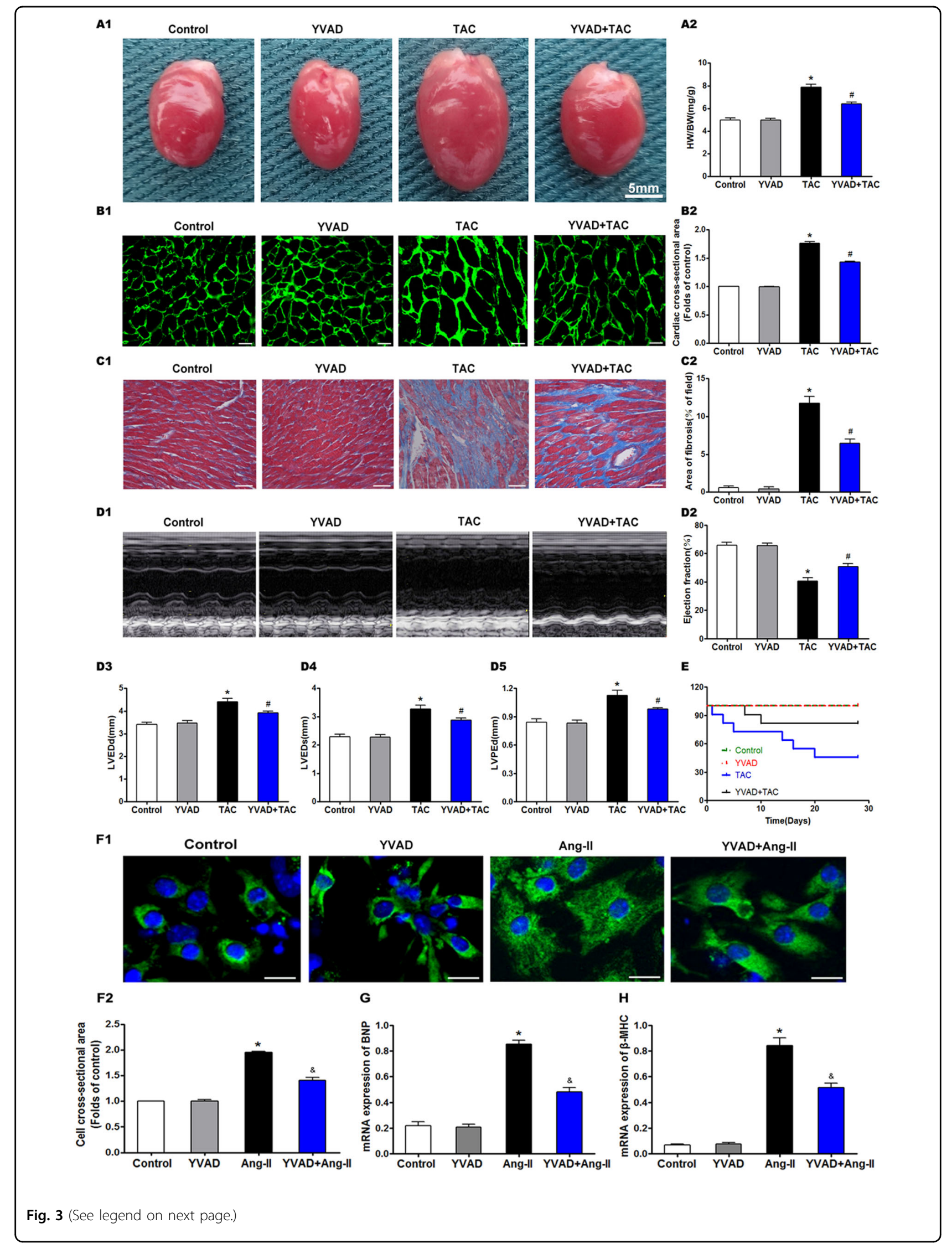


(see figure on previous page)

Fig. 3 Caspase-1 inhibition attenuates overload-stimulated cardiac hypertrophy. A1 Representative pictures showing mouse hearts in the indicated groups. A2 Proportion of heart to body weight in the indicated groups. B1 Representative images showing the cross-sectional area of cardiomyocytes of the left ventricle in the indicated groups. Heart tissues were immune-stained with WGA. Green-anti-WGA. One hundred cells were randomly selected from five sections of each heart. Scale bars: $20 \mu \mathrm{m}$. B2 Quantification of the cross-sectional area of cardiomyocytes of the left ventricle in the indicated groups. C1 Representative images showing the myocardial fibrosis, assessed using Masson trichrome staining in the indicated groups. Blue areas represent fibrotic staining. Fibrosis was quantified by five whole LV sections for each heart. Scale bars: $50 \mu \mathrm{m}$. C2 Quantification of the myocardial fibrosis in the indicated groups. The cardiac structure and function of mice were assessed by echocardiography 4 weeks after TAC. D1 Representative images of M-mode echocardiography for mouse hearts. Quantitative analysis of left ventricular EF (D2), LVEDd (D3), LVEDs (D4), and LVPWd (D5). E Survival curves of mice using Kaplan and Meier. Characteristic images (F1) and measurement (F2) of the cell surface area of cardiomyocytes. Cardiomyocytes were stained with cardiomyocyte marker a-actinin (green fluorescence). Nuclei were marked with DAPI (blue fluorescence), Scale bars: $20 \mu \mathrm{m}$. In total, 100 cells were selected on random and assessed for surface area for each cohort from six independent trials. Real-time PCR of mRNA expression of cardiac hypertrophy markers BNP $(\mathbf{G})$ and $\beta-M H C(\mathbf{H})$. Results are presented as the mean \pm SEM, $n=6 .{ }^{*} P<0.01$ compared to control, ${ }^{\#} P<0.01$ compared to TAC group, ${ }^{\circledR} P<0.01$ compared to Ang-ll group.

improve the symptoms of overload-stimulated cardiac hypertrophy and heart failure.

\section{Overexpression of NLRP3 mitigates the cardioprotective effects of irisin}

To evaluate the role of exogenous irisin in controlling NLRP3-stimulated inflammation, we treated ectopic NLRP3-overexpressing cardiomyocytes with irisin. The high levels of NLRP3 reversed the irisin-mediated suppression of ASC, cleaved caspase-1, and GSDMD-N (Fig. 6A), and also increased the number of PI-positive cells (Fig. 6B), cardiomyocyte surface area (Fig. 6C), and the BNP and $\beta$-MHC levels (Fig. 6D). To summarize, NLRP3 inflammasome-mediated signaling influences the antipyroptotic and antihypertrophic effects of irisin.

\section{Discussion}

We found that pyroptosis is associated with cardiac hypertrophy and the cardioprotective effects of irisin are mediated by inhibiting pyroptosis. Moreover, we also confirmed that the pressure overload-induced pyroptosis is driven by the NLRP3 inflammasome, and inhibiting the pyroptotic cascade alleviates cardiac hypertrophy. Finally, the cardioprotective effects of irisin were abrogated by NLRP3 overexpression, which underscored that NLRP3mediated pyroptosis is the main target of irisin.

Pyroptosis refers to a form of pro-inflammatory cell death that is contingent on caspase- 1 stimulation ${ }^{26,27}$ and is the pathological basis of numerous disorders. For instance, nicotine promotes atherosclerosis by inducing pyroptosis of endothelial cells ${ }^{28}$. In addition, endothelial pyroptosis is also prevalent during endotoxemia-stimulated lung damage ${ }^{29}$. The close relationship between heart disease and pyroptosis has also been demonstrated in previous studies ${ }^{30-32}$. Nevertheless, the molecular mechanisms underlying pyroptosis in cardiac hypertrophy were not clear. We established in vivo and in vitro models of cardiac hypertrophy via TAC and Ang-II treatment, respectively, and observed a significant increase in pyroptotic cells, along with upregulation in IL-1 $\beta$, cleaved caspase-1, and GSDMD-N. These findings clearly indicated that pyroptosis has a pathological role in cardiac hypertrophy.

Irisin is abundant in the adipose tissues, skeletal muscles, and heart ${ }^{33,34}$. A previous study showed that irisin levels were decreased in cardiac tissues and plasma after TAC, as well as in Ang-II-stimulated cardiomyocytes ${ }^{35}$. Since the cardiac tissues are the main source of $\operatorname{irisin}^{36}$, loss of cardiomyocytes may contribute to a reduction in irisin after hypertrophy. There is evidence that exogenous irisin can reverse NLRP3-stimulated inflammation ${ }^{37}$, indicating that exogenous supplementation of recombinant irisin may partially reverse cardiac hypertrophy. We found that irisin normalized the aberrantly high levels of IL- $1 \beta$, cleaved caspase-1, and GSDMD-N after TAC or Ang-II treatment. In the mouse model, irisin mitigated TAC-induced cardiac hypertrophy and fibrosis, as indicated by reduced HW/BW, smaller cardiomyocytes, and improved echocardiography parameters $(\mathrm{EF})$. Thus, irisin can ameliorate heart failure and cardiac hypertrophy by inhibiting pyroptosis.

Caspase- 1 activation by the inflammasome complex leads to the cleavage and secretion of pro-inflammatory IL-1 $\beta^{38}$. Inflammasomes can trigger both caspase-1dependent and -independent pyroptosis following tissue injury $^{39-42}$. Our findings indicated that NLRP3/caspase-1dependent pyroptosis has a pathological role in myocardial hypertrophy. Targeted inhibition of NLRP3 by CRID3 and gene silencing blocked caspase-1-dependent pyroptosis in cardiomyocytes and alleviated the signs of cardiac hypertrophy. Furthermore, irisin inhibited NLRP3-mediated pyroptosis, and its cardioprotective effects were abrogated by overexpression of NLRP3.

To summarize, NLRP3-mediated pyroptosis has a vital function in cardiac hypertrophy, and irisin can mitigate cardiac hypertrophy and heart failure by inhibiting the pyroptosis cascade.

\section{Materials and methods Mouse model of cardiac hypertrophy}

Cardiac hypertrophy was established in 4-month-old male C57BL/6J mice using transverse aortic constriction 


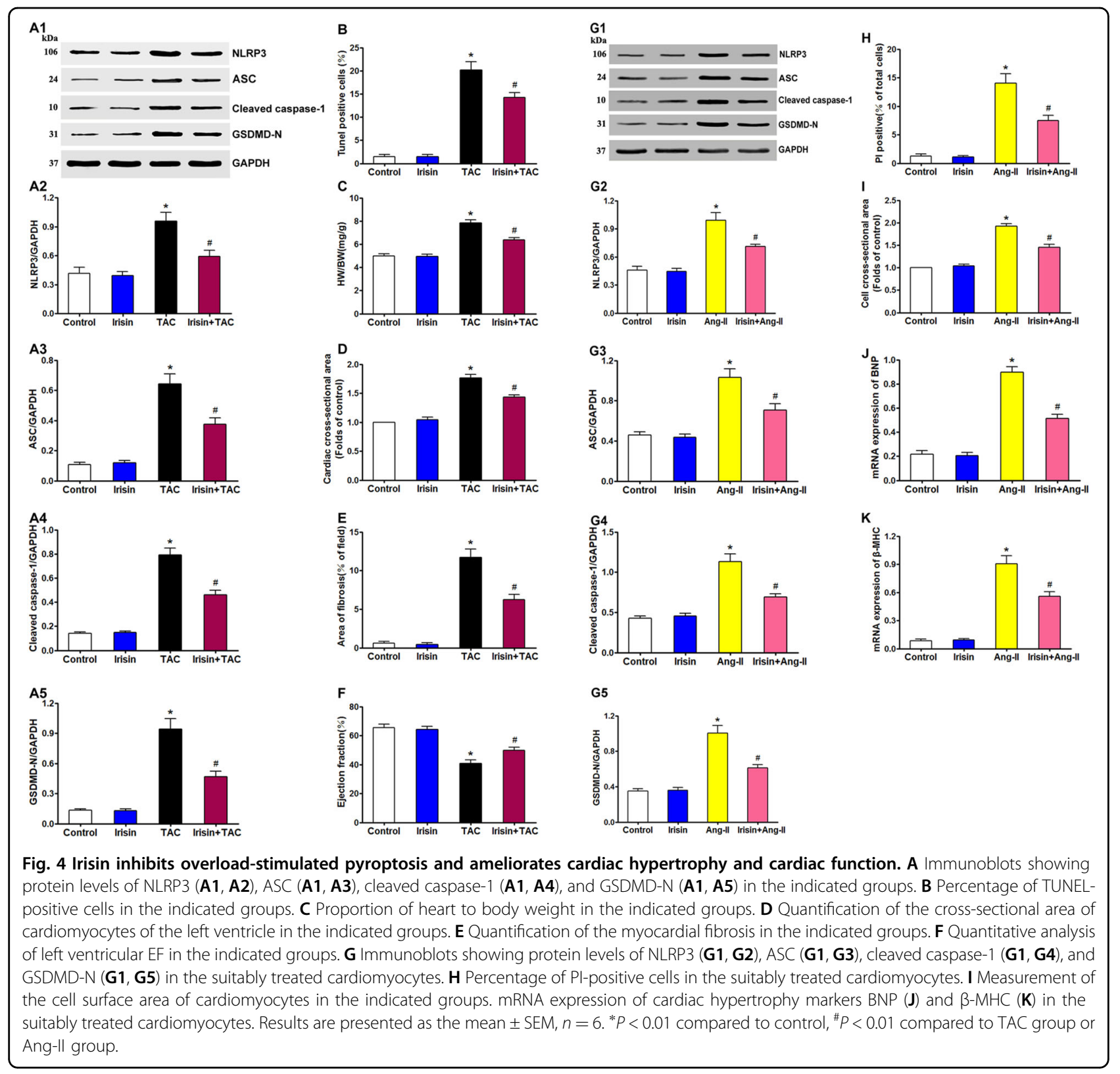

$(\mathrm{TAC})^{43}$. The mice were anesthetized and placed in a supine position, and a midline cervical incision was made to expose the trachea. After opening the chest cavity, the transverse aorta was sutured using $7-0$ nylon thread, connected using a blunt 27-gauge needle, and then detached. The sham-operated controls underwent the same procedure without aortic ligation. The chest was then sutured, and the animals were ventilated until they could breathe on their own. The mice were intravenously injected with PBS (control) or recombinant irisin $(2 \mu \mathrm{g} / \mathrm{kg} /$ week, Phoenix Pharmaceuticals, Inc., USA ${ }^{44}$ at $0,1,2$, and 3 weeks after TAC, and euthanized after 4 weeks. The cardiac tissues were resected for further analysis. All experimental procedures were conducted as per the guidelines for the Animal Care and Use Committee of North Sichuan Medical College, China, and approved by the Medical Ethics Committee.

\section{In vitro model of cardiomyocyte hypertrophy}

Primary cardiomyocytes were obtained from 1- to 3day-old neonatal mice as previously described ${ }^{45}$, and cultured for $12 \mathrm{~h}$ in serum-free DMEM. According to published protocols ${ }^{44}$, the cardiomyocytes were treated with PBS or Ang-II $(1 \mathrm{ng} / \mathrm{ml})$ with/out irisin $(100 \mathrm{ng} / \mathrm{ml})$ for $0,0.5,1,24$, and $48 \mathrm{~h}$. The serum-free medium containing Ang II was changed every $24 \mathrm{~h}$, and the cells were harvested for further analysis. 


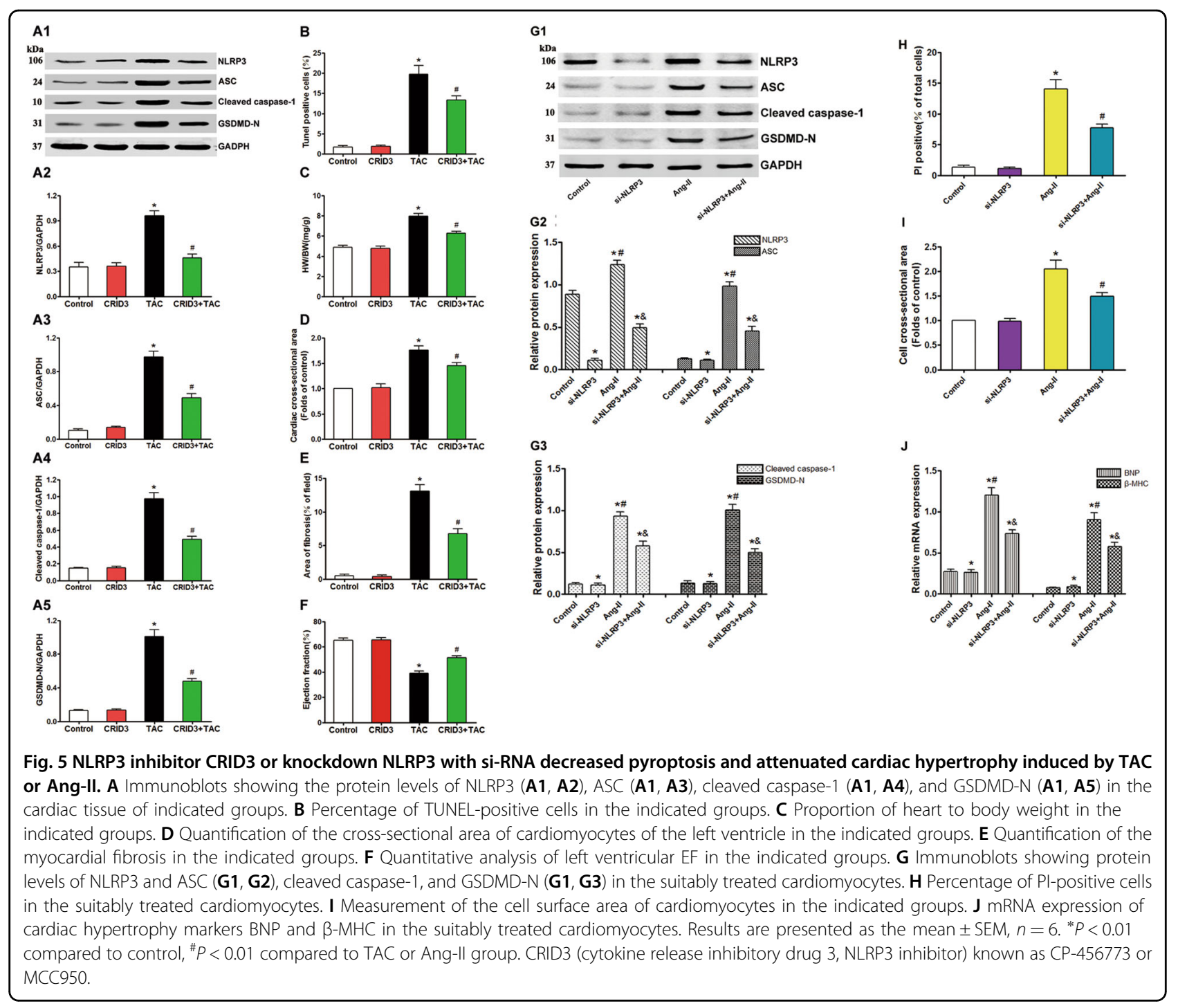

\section{Echocardiography}

The mice were anesthetized with a combination of oxygen $(2 \mathrm{~L} / \mathrm{min})$ and isoflurane $(2 \%)$ and subjected to transthoracic echocardiography at stipulated time points using GE vivid 8-dimension ultrasound. The heart was observed on the short axis among two papillary muscles, and every dimension was acquired using M-mode by taking the mean of three successive heartbeats. The EF\% (LV ejection fraction), LVEDs (left ventricular end-systolic diameter), LVEDd (left ventricular end-diastolic dimension), and LVPWd (left ventricular diastolic posterior wall thickness) were measured by the same technician, and the average of six consecutive heart cycles was calculated.

\section{Histology and immunohistochemistry}

The hearts of the model and sham-operated mice were resected 4 weeks post TAC, weighed, perfused with PBS, fixed with $4 \%(\mathrm{w} / \mathrm{v})$ paraformaldehyde, and paraffin- embedded or snap-frozen in liquid nitrogen. The paraffinembedded tissues were cut into $4-\mu \mathrm{m}$-thick sections. After blocking with goat serum at room temperature for $30 \mathrm{~min}$, the sections were immunostained with anti-wheat germ agglutinin (WGA) antibody to assess the cross-sectional area of the cardiomyocytes ${ }^{46}$. The degree of fibrosis was assessed by Masson trichrome staining of the left ventricular wall sections. Five random high-powered fields were examined per section in a blinded manner. Images were analyzed using the ImageJ program.

\section{Immunocytochemistry}

Cardiomyocytes were cultured on coverslips in vitro as appropriate and fixed in $4 \%$ paraformaldehyde at room temperature for $20 \mathrm{~min}$. After permeabilizing in $0.1 \%$ Triton X-100 for $10 \mathrm{~min}$, the cells were blocked with 3\% BSA for $30 \mathrm{~min}$ and then incubated with the anti- $\alpha$-actinin antibody (1:100, Abcam). The cells were then probed 


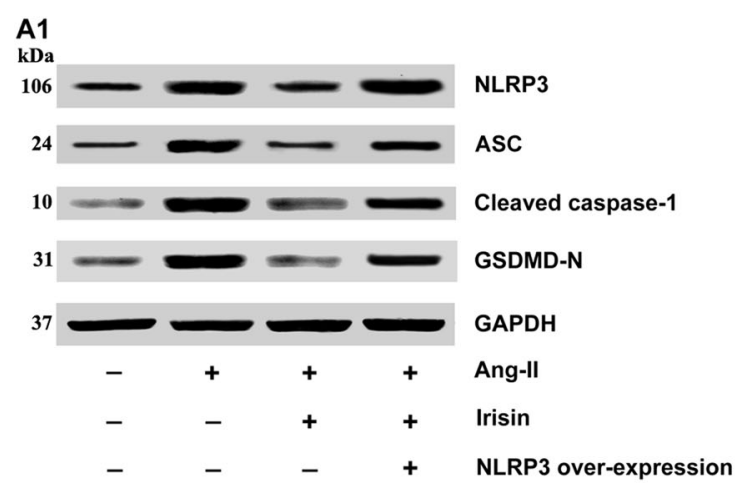

\section{B}

A2

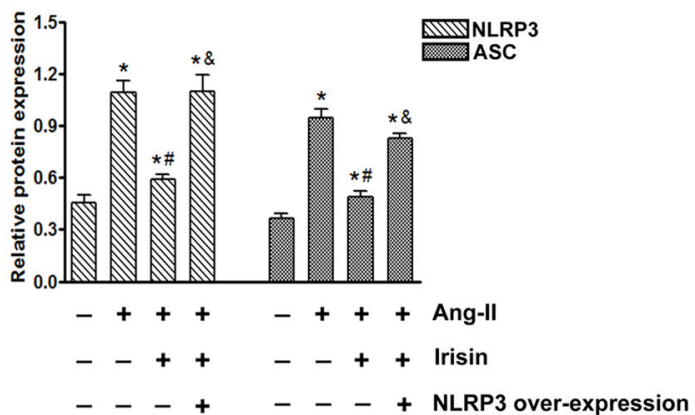

A3
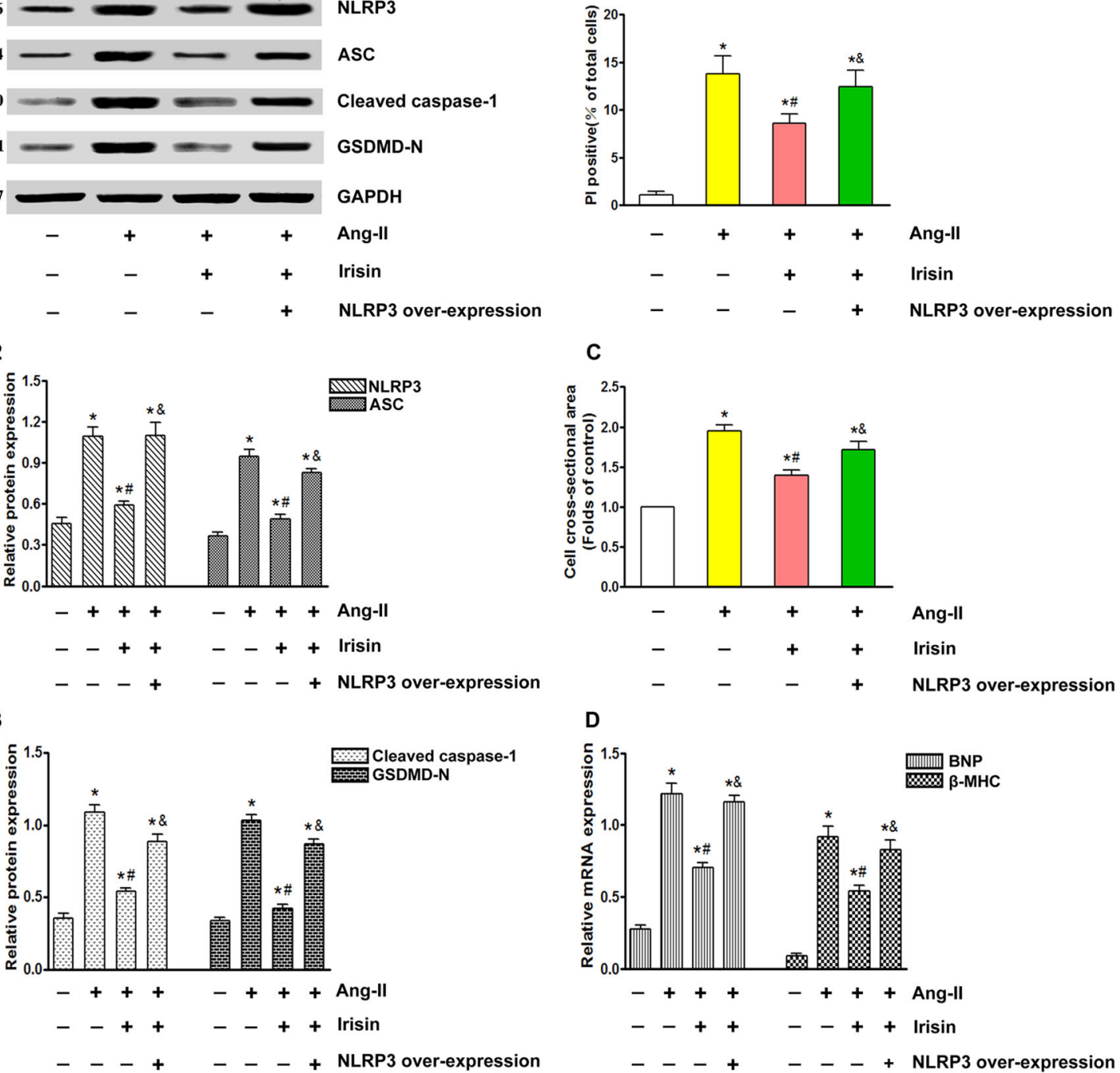

Fig. 6 Overexpression of NLRP3 reversed irisin-mediated antipyroptosis and anticardiac hypertrophy effects. A Immunoblots showing protein levels of NLRP3 and ASC (A1, A2), cleaved caspase-1, and GSDMD-N (A1, A3) in the suitably treated cardiomyocytes. $\mathbf{B}$ Percentage of Plpositive cells in the suitably treated cardiomyocytes. C Measurement of the cell surface area of cardiomyocytes in the indicated groups. $\mathbf{D}$ mRNA expression of cardiac hypertrophy markers BNP and $\beta-\mathrm{MHC}$ in the suitably treated cardiomyocytes. Results are presented as the mean \pm SEM, $n=6$. ${ }^{*} P<0.01$ compared to control, ${ }^{\#} P<0.01$ compared to Ang-\|l group, ${ }^{\circledR} P<0.01$ versus Ang- $\|$ + Irisin group.

with Alexa Fluor 647-conjugated secondary antibody at room temperature for $60 \mathrm{~min}$, and the nuclei were counterstained with DAPI. The stained cells were observed under a fluorescence microscope (Nikon, Japan), and the surface area of each cardiomyocyte was quantified using ImageJ. Five independent zones were selected per slide and the area was quantified in a blinded manner and normalized to the control group.

\section{TUNEL assay}

TUNEL assay was performed as described previously ${ }^{47}$ using the in situ cell death detection system (Roche, USA). The cardiomyocytes were stained using the sarcomeric tropomyosin antibody and the nuclei were counterstained with DAPI. The cells were observed under a Nikon fluorescence microscope (Nikon, Tokyo, Japan), and the average fluorescence intensity was assessed with the ImageJ software. TUNEL-positive nuclei were counted in 15 nonoverlapping regions, and the percentage was calculated relative to the total number of cells per field. The experiment was repeated six times in a blinded manner.

\section{Propidium iodide (PI) staining}

The suitably treated cells were washed with PBS and stained with DAPI $(5 \mathrm{ml})$ and PI $(5 \mathrm{ml})$ for $20 \mathrm{~min}$ in the 
dark at $37^{\circ} \mathrm{C}$. The PI-positive cells were counted per 200 cells in three random fields under a fluorescence microscope (Nikon, Tokyo, Japan), and the average fluorescence intensity was assessed with the ImageJ software.

\section{Real-time RT-PCR}

RNA was extracted from the cardiac tissues using the RNAiso plus kit (TAKARA). The transcript levels of brain natriuretic peptide (BNP) and $\beta$-myosin heavy chain $(\beta-\mathrm{MHC})$ were analyzed by RT-PCR using the TOYOBO kit and the iQ5 Real-Time PCR Detection System (BioRad, Hercules, CA) as per instructions. The SYBR1 premix Ex TaqTM II was used according to the recommended guidelines (TaKaRa Biotechnology Co. Ltd., Tokyo, Japan), and GAPDH was the internal standard. The melting curves and values were assessed with the BioRAD software. The relative mRNA level was quantified using the comparative cycle threshold method. Each sample was analyzed at least thrice.

\section{Western blotting}

Western blotting was conducted as described ${ }^{48}$ using antibodies against ASC (1:1000, Abcam, ab151700), GSDMD-N (1:200, Abcam, ab215203), NLRP3 (1:200, Abcam, ab270449), IL-1 $\beta$ (1:1000, Abcam, ab200478), cleaved caspase-1 (1:200, Abcam, ab238972), and the internal control GAPDH (1:1000, Abcam, ab8245). The protein bands were quantified using the Odyssey color infrared laser scanning imager (LI-COR, Lincoln, NE).

\section{Statistical analyses}

All results were presented as mean \pm SEM of five independent experiments. GraphPad Prism 5.0 software (San Diego, CA, USA) was used for statistical analysis. Oneway factorial ANOVA was used to compare multiple groups, and the Student's $t$ test for pairwise comparison. The Kaplan-Meier method was used for survival analysis. $P<0.05$ was considered statistical significance.

\section{Acknowledgements}

This study was granted support using the National Natural Science Foundation of China (81600232), Science and Technology Strategic Special Cooperation between City and University in NanChong (18SXHZ0458; 18SXHZ0532; 18SXHZ0545), and Research project foundation of Affiliated Hospital of North Sichuan Medical College (2020ZD005; 2021ZK002).

\section{Author details}

${ }^{1}$ Department of Cardiology, Affiliated Hospital of North Sichuan Medical College, 637000 Nanchong, P.R. China. ${ }^{2}$ CSSD, Affiliated Hospital of North Sichuan Medical College, 637000 Nanchong, P.R. China. ${ }^{3}$ Ophthalmology Department, Affiliated Hospital of North Sichuan Medical College, 637000 Nanchong, P.R. China. ${ }^{4}$ Anesthesiology Department, Affiliated Hospital of North Sichuan Medical College, 637000 Nanchong, P.R. China. ${ }^{5}$ Department of Respiratory, Affiliated Hospital of North Sichuan Medical College, 637000 Nanchong, P.R. China

\section{Data availability}

The data used to support the findings of this study are included in the paper.
Conflict of interest

The authors declare no competing interests.

\section{Publisher's note}

Springer Nature remains neutral with regard to jurisdictional claims in published maps and institutional affiliations.

Received: 24 November 2020 Revised: 31 January 2021 Accepted: 14 February 2021

Published online: 15 March 2021

\section{References}

1. Curtis, L. H. et al. Early and long-term outcomes of heart failure in elderly persons, 2001-2005. Arch. Intern. Med. 168, 2481-2488 (2008).

2. Gao, L. et al. Novel role for caspase-activated DNase in the regulation of pathological cardiac hypertrophy. Hypertension 65, 871-881 (2015).

3. Haque, Z. K. \& Wang, D. Z. How cardiomyocytes sense pathophysiological stresses for cardiac remodeling. Cell. Mol. Life Sci. 74, 983-1000 (2017).

4. Kallikourdis, M. et al. T cell costimulation blockade blunts pressure overloadinduced heart failure. Nat. Commun. 8, 14680 (2017).

5. Tham, Y. K., Bernardo, B. C., Ooi, J. Y., Weeks, K. L. \& McMullen, J. R. Pathophysiology of cardiac hypertrophy and heart failure: signaling pathways and novel therapeutic targets. Arch. Toxicol. 89, 1401-1438 (2015).

6. Heger, J., Schulz, R. \& Euler, G. Molecular switches under TGFbeta signalling during progression from cardiac hypertrophy to heart failure. Br. J. Pharmacol. 173, 3-14 (2016)

7. Turner, N. A. Inflammatory and fibrotic responses of cardiac fibroblasts to myocardial damage associated molecular patterns (DAMPs). J. Mol. Cell. Cardiol. 94, 189-200 (2016).

8. Wu, Q. Q. et al. Mechanisms contributing to cardiac remodelling. Clin. Sci. 131, 2319-2345 (2017)

9. Zhou, L. et al. Cardioprotective role of myeloid-derived suppressor cells in heart failure. Circulation 138, 181-197 (2018).

10. Jiang, D., Chen, S., Sun, R., Zhang, X. \& Wang, D. The NLRP3 inflammasome: role in metabolic disorders and regulation by metabolic pathways. Cancer Lett. 419, 8-19 (2018).

11. Dempsey, C. et al. Inhibiting the NLRP3 inflammasome with MCC950 promotes non-phlogistic clearance of amyloid-beta and cognitive function in APP/PS1 mice. Brain, Behav., Immun. 61, 306-316 (2017).

12. $\mathrm{Xu}$, J. et al. Macrophage endocytosis of high-mobility group box 1 triggers pyroptosis. Cell Death Differ. 21, 1229-1239 (2014).

13. Jorgensen, I. \& Miao, E. A. Pyroptotic cell death defends against intracellular pathogens. Immunological Rev. 265, 130-142 (2015).

14. Zhou, W. et al. NLRP3: a novel mediator in cardiovascular disease. J. Immunol. Res. 2018, 5702103 (2018).

15. Bostrom, P. et al. A PGC1-alpha-dependent myokine that drives brown-fat-like development of white fat and thermogenesis. Nature 481, 463-468 (2012).

16. Zhu, D. et al. Irisin improves endothelial function in type 2 diabetes through reducing oxidative/nitrative stresses. J. Mol. Cell. Cardiol. 87, 138-147 (2015).

17. Hou, N. et al. Irisin regulates heme oxygenase-1/adiponectin axis in perivascular adipose tissue and improves endothelial dysfunction in diet-induced obese mice. Cell. Physiol. Biochem. 42, 603-614 (2017).

18. Zhang, Y. et al. Protective effect of irisin on atherosclerosis via suppressing oxidized low density lipoprotein induced vascular inflammation and endothelial dysfunction. PLOS ONE 11, e0158038 (2016).

19. Fu, J., Han, Y., Wang, J., Jose, P. A. \& Zeng, C. Irisin lowered blood pressure by augmenting acetylcholine-mediated vasodilation via AMPK-Akt-eNOS-NO signal pathway in the spontaneously hypertensive rat. J. Am. Soc. Hypertension 10(Suppl 1), e4 (2016).

20. Wang, $H$. et al. Irisin plays a pivotal role to protect the heart against ischemia and reperfusion injury. J. Cell. Physiol. 232, 3775-3785 (2017).

21. Tan, C. C. et al. NLRP1 inflammasome is activated in patients with medial temporal lobe epilepsy and contributes to neuronal pyroptosis in amygdala kindling-induced rat model. J. Neuroinflam. 12, 18 (2015).

22. Tang, M. et al. Bradykinin B2 receptors play a neuroprotective role in Hypoxia/ reoxygenation injury related to pyroptosis pathway. Curr. Neurovasc. Res. 15, 138-144 (2018). 
23. An, N. et al. Regulatory mechanisms of the NLRP3 inflammasome, a nove immune-inflammatory marker in cardiovascular diseases. Front. Immunol. 10 1592 (2019).

24. Kelley, N., Jeltema, D., Duan, Y. \& He, Y. The NLRP3 inflammasome: an overview of mechanisms of activation and regulation. Int. J. Mol. Sci. 20, 3328 (2019).

25. Pellegrini, C., Fornai, M., Antonioli, L., Blandizzi, C. \& Calderone, V. Phytochemicals as novel therapeutic strategies for NLRP3 inflammasome-related neurological, metabolic, and inflammatory diseases. Int. J. Mol. Sci. 20, 2876 (2019).

26. Bergsbaken, T., Fink, S. L. \& Cookson, B. T. Pyroptosis: host cell death and inflammation. Nat. Rev. Microbiol. 7, 99-109 (2009).

27. Doitsh, G. et al. Cell death by pyroptosis drives CD4 T-cell depletion in HIV-1 infection. Nature 505, 509-514 (2014).

28. $\mathrm{Wu}, \mathrm{X}$. et al. Nicotine promotes atherosclerosis via ROS-NLRP3-mediated endothelial cell pyroptosis. Cell Death Dis. 9, 171 (2018).

29. Cheng, K. T. et al. Caspase-11-mediated endothelial pyroptosis underlies endotoxemia-induced lung injury. J. Clin. Investig. 127, 4124-4135 (2017).

30. Zhaolin, Z., Guohua, L., Shiyuan, W. \& Zuo, W. Role of pyroptosis in cardiovascular disease. Cell Prolif. 52, e12563 (2019).

31. Ye, B. et al. Emodin alleviates myocardial ischemia/reperfusion injury by inhibiting gasdermin D-mediated pyroptosis in cardiomyocytes. Drug Des., Dev. Ther. 13, 975-990 (2019)

32. Audia, J. P. et al. Caspase-1 inhibition by VX-765 administered at reperfusion in P2Y12 receptor antagonist-treated rats provides long-term reduction in myocardial infarct size and preservation of ventricular function. Basic Res. Cardiol. 113, 32 (2018)

33. Colaianni, G., Cinti, S., Colucci, S. \& Grano, M. Irisin and musculoskeletal health. Ann. N. Y. Acad. Sci. 1402, 5-9 (2017).

34. Perakakis, N. et al. Physiology and role of irisin in glucose homeostasis. Nat. Rev. Endocrinol. 13, 324-337 (2017).

35. Li, R. L. et al. Irisin alleviates pressure overload-induced cardiac hypertrophy by inducing protective autophagy via mTOR-independent activation of the AMPK-ULK1 pathway. J. Mol. Cell. Cardiol. 121, 242-255 (2018).
36. Aydin, S. et al. Cardiac, skeletal muscle and serum irisin responses to with or without water exercise in young and old male rats: cardiac muscle produces more irisin than skeletal muscle. Peptides 52, 68-73 (2014).

37. Peng, J. et al. Irisin protects against neuronal injury induced by oxygenglucose deprivation in part depends on the inhibition of ROS-NLRP3 inflammatory signaling pathway. Mol. Immunol. 91, 185-194 (2017).

38. Gaidt, M. M. \& Hornung, V. Pore formation by GSDMD is the effector mechanism of pyroptosis. EMBO J. 35, 2167-2169 (2016).

39. Kovarova, M. et al. NLRP1-dependent pyroptosis leads to acute lung injury and morbidity in mice. J. Immunol. 189, 2006-2016 (2012).

40. Pronin, A. et al. Inflammasome activation induces pyroptosis in the retina exposed to ocular hypertension injury. Front. Mol. Neurosci. 12, 36 (2019).

41. Xue, Z. et al. miR-21 promotes NLRP3 inflammasome activation to mediate pyroptosis and endotoxic shock. Cell Death Dis. 10, 461 (2019).

42. Yang, J. R. et al. Ischemia-reperfusion induces renal tubule pyroptosis via the CHOP-caspase-11 pathway. Am. J. Physiol. Ren. Physiol. 306, F75-F84 (2014)

43. Wang, Z. et al. The long noncoding RNA Chaer defines an epigenetic checkpoint in cardiac hypertrophy. Nat. Med. 22, 1131-1139 (2016).

44. $Y u, Q$. et al. FNDC5//risin inhibits pathological cardiac hypertrophy. Clin. Sci. 133, 611-627 (2019).

45. Yue, R. et al. Lycopene protects against hypoxia/reoxygenation-induced apoptosis by preventing mitochondrial dysfunction in primary neonatal mouse cardiomyocytes. PLoS ONE 7, e50778 (2012).

46. Pedrozo, Z. et al. Polycystin-1 is a cardiomyocyte mechanosensor that governs L-type Ca2+ channel protein stability. Circulation 131, 2131-2142 (2015).

47. Yue, R. et al. Mitochondrial DNA oxidative damage contributes to cardiomyocyte ischemia/reperfusion-injury in rats: cardioprotective role of lycopene. J. Cell. Physiol. 230, 2128-2141 (2015).

48. Luo, T. et al. PD150606 protects against ischemia/reperfusion injury by preventing mu-calpain-induced mitochondrial apoptosis. Arch. Biochem. Biophys. https://doi.org/10.1016/j.abb.2015.06.005 (2015) 